\title{
A putative ariadne-like E3 ubiquitin ligase (PAUL) that interacts with the muscle-specific kinase (MuSK).
}

\section{Bromann, Paul Andrew}

2004-01

Bromann , P A , Weiner , J A , Apel , E D , Lewis , R M \& Sanes , J R 2004 , ' A putative ariadne-like E3 ubiquitin ligase (PAUL) that interacts with the muscle-specific kinase (MuSK). ' , Gene expression patterns, vol. 4 , no. 1 , pp. 77-84 . < http://www.ncbi.nlm.nih.gov/pubmed/14678832 >

http://hdl.handle.net/10138/33906

acceptedVersion

Downloaded from Helda, University of Helsinki institutional repository.

This is an electronic reprint of the original article.

This reprint may differ from the original in pagination and typographic detail.

Please cite the original version. 


\title{
A putative ariadne-like E3 ubiquitin ligase (PAUL) that interacts with the muscle-specific kinase (MuSK)
}

\author{
Paul A. Bromann ${ }^{1}$, Joshua A. Weiner, Elizabeth D. Apel ${ }^{2}$, Renate M. Lewis, Joshua R. Sanes* \\ Department of Anatomy and Neurobiology, Washington University Medical School, 660 South Euclid, St Louis, MO 63110, USA
}

Received 1 May 2003; received in revised form 15 July 2003; accepted 16 July 2003

\begin{abstract}
Formation of the postsynaptic membrane at the skeletal neuromuscular junction (NMJ) requires activation of the muscle-specific receptor tyrosine kinase (MuSK). Few intracellular mediators or modulators of MuSK actions are known. E3 ubiquitin ligases may serve this role, because activities of several receptor tyrosine kinases, G-protein-coupled receptors and channels are modulated by ubiquitination. Here, we report identification of a putative Ariadne-like ubiquitin ligase (PAUL) that binds to the cytoplasmic domain of MuSK. PAUL is expressed in numerous tissues of developing and adult mice, and is present at NMJs in muscle fibers but is not confined to them.
\end{abstract}

(C) 2003 Elsevier B.V. All rights reserved.

Keywords: Synapse; Neuromuscular junction; Acetylcholine receptors; Muscle-specific receptor tyrosine kinase; Ubiquitination; E3 ubiquitin ligase; Ariadne; Yeast-two-hybrid

Much of what is known about the mechanisms of synaptic development comes from the study of the vertebrate neuromuscular junction (NMJ) (Sanes and Lichtman, 1999). A well-studied aspect of this process is the clustering of acetylcholine receptors (AChRs) in the postsynaptic membrane. AChR clustering is promoted and/or stabilized by the presynaptic protein agrin; agrin, in turn, signals by activating the muscle specific receptor tyrosine kinase (MuSK) (McMahan, 1990; Glass et al., 1996; Sanes and Lichtman, 2001). Little is known, however, about how activation of MuSK leads to AChR clusters, or how MuSK levels and activity are regulated.

An intriguing system in this regard is E3-dependent ubiquitination, which has recently been shown to serve as a regulatory mechanism for signaling and trafficking of several receptors. For example, the adaptor protein $\mathrm{Cbl}$ has been shown to ubiquitinate and thereby regulate receptor tyrosine kinase signaling through its E3 activity (Pride et al., 2002). In addition, ßarrestin-dependent ubiquitination by an E3, Mdm2, attenuates adrenergic

\footnotetext{
* Corresponding author. Tel.: + 1-314-362-2507; fax: + 1-314-747-1150.

E-mail address: sanesj@pcg.wustl.edu (J.R. Sanes).

1 Present address: Van Andel Research Institute, Grand Rapids, MI, USA.

2 Present address: ICOS Corporation, Seattle, WA, USA.
}

receptor signaling (Shenoy et al., 2001). Related mechanisms may be involved in neuromuscular development. Indeed, AChR trafficking in cultured myotubes is affected by ubiquitination (Keller et al., 2001) and ubiquitin ligases play key roles in muscle growth and atrophy (Glass, 2003). Moreover, the ubiquitin pathway has recently been shown to regulate NMJ development in Drosophila (DiAntonio et al., 2000; Hegde and DiAntonio, 2002) and synaptic composition at mammalian synapses (Ehlers, 2003). These data raise the possibility that E3 ubiquitin ligases could play a role in MuSK signaling and regulation of neuromuscular development in vertebrates. Consistent with this idea, we have found, and describe here, a putative Ariadne-like ubiquitin ligase that binds directly to MuSK.

\section{Results and discussion}

\subsection{Identification and structure of PAUL}

To identify components of the MuSK signaling pathway, we employed the yeast two-hybrid system, using the cytoplasmic domain of MuSK as bait (Apel et al., 2000). Of 61 positive clones obtained from an E17 mouse cDNA library, 12 encoded an 875 aa fragment of a novel protein; 
a 13th encoded an overlapping fragment of the same protein. Rapid amplification of cDNA ends (RACE) was used to obtain additional sequence, leading to assembly of a $3.8 \mathrm{~kb}$ sequence that encoded a single 1067 aa open reading frame (Fig. 1a), along with $3^{\prime}$ and $5^{\prime}$ UTR (Genbank accession number AY283085).

Analysis of the deduced amino acid sequence showed that it contains, from amino- to carboxy-terminal, a cysteine-rich zinc finger (BBOX) a serine-rich region, a Ran bindingprotein zinc finger domain (zf-RanBP), an ubiquitin association (UBA) domain, a Really Interesting New Gene (RING) domain, an In-Between RING (IBR) domain, and a second RING domain (Fig. 1a). Both BBOX and zf-RanBP domains are believed to be involved in mediating proteinprotein interactions. RING and IBR domains are most often found in a large and diverse family of E3 ubiquitin ligases,

(a)

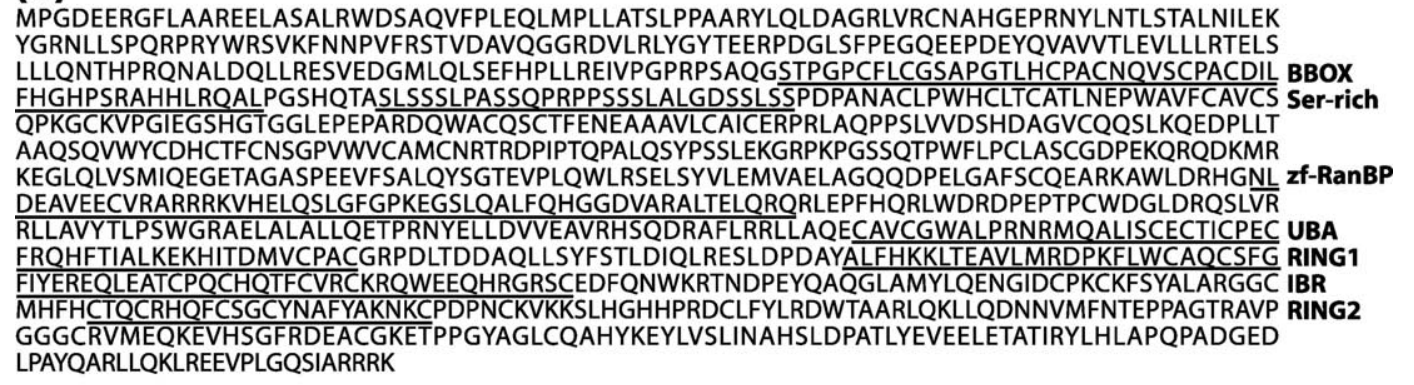

(b)
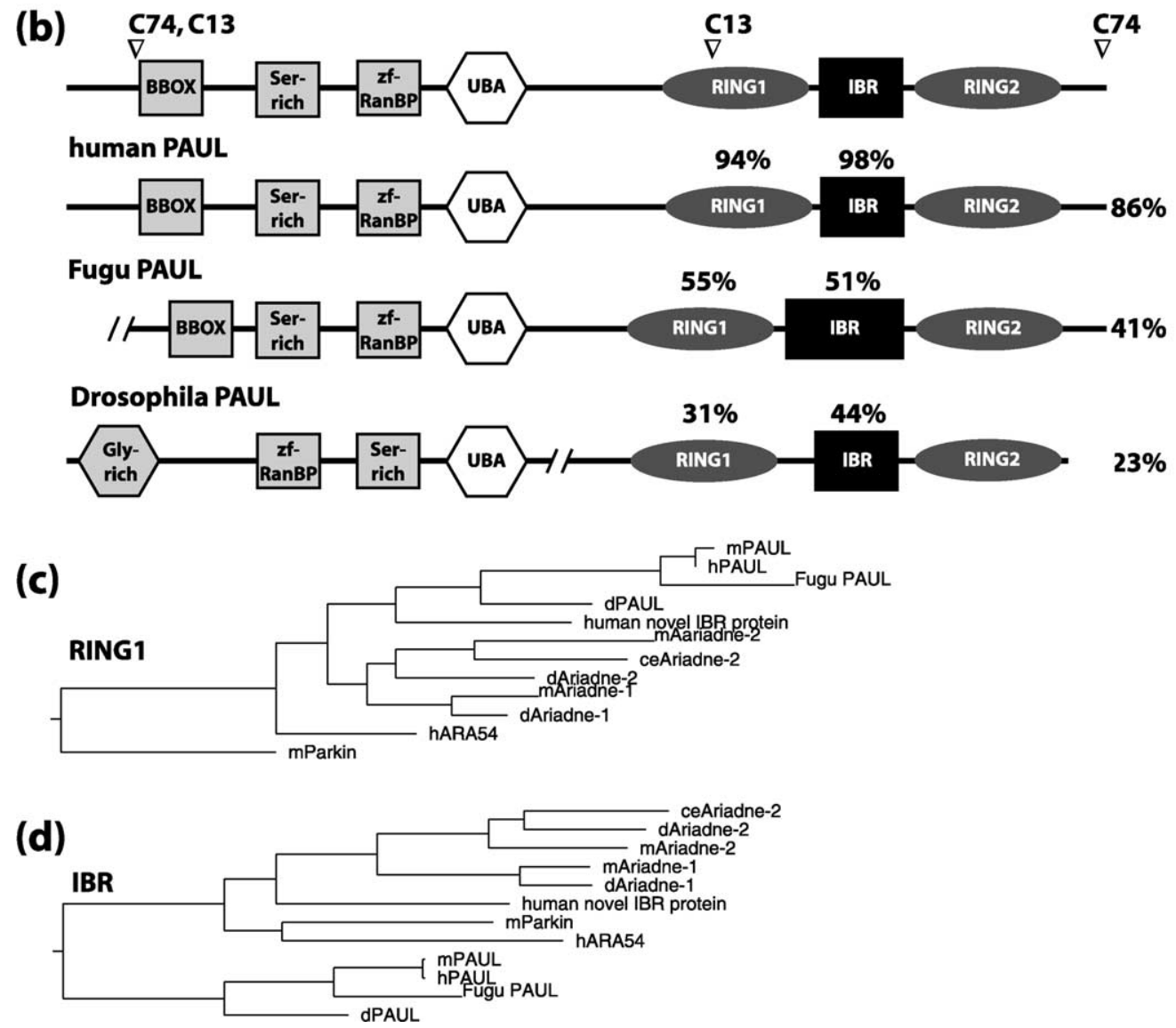

Fig. 1. Primary sequence of PAUL and comparison with related proteins. (a) Deduced amino acid sequence of PAUL. Recognizable domains are underlined. BBOX, B box domain; Ser-rich, serine-rich domain; zf-RanBP, Ran binding-protein zinc finger domain; UBA, UBA domain; RING, RING domain; IBR, IBR domain. (b) Domain structure of mouse PAUL and its relationship to putative orthologues from human, Fugu and Drosophila. Figures at right indicate percent amino acid sequence identity of the whole proteins with mouse PAUL; figures between lines indicate similarity of the RING1 and IBR domains. Arrowheads labeled C13 and C74 indicate termini of two classes of clones isolated by yeast two-hybrid screening. (c,d) Relationship of RING1 and IBR domains of PAUL with corresponding domains in other ariadne-like proteins that share the RBR signature (Marin and Ferrus, 2002). Human (h), fugu, and Drosophila (d) orthologues are most similar to mouse (m) PAUL; more distant relatives are Ariadne-subfamily proteins and Parkin. Genbank accession numbers are in Section 2. 
including Ariadne (see below), which catalyze the ligation of activated ubiquitin to substrate proteins (Joazeiro and Weissman, 2000). The UBA domain is found in several proteins involved in ubiquitin signaling, including some E3 ubiquitin ligases (Hofmann and Bucher, 1996). Based on these signature motifs, we call the novel protein Putative Ariadne-like Ubiquitin Ligase or PAUL.

Searches of public and Celera databases identified a human cDNA, FLJ10111, which is $86 \%$ identical to mouse PAUL (Fig. 1b) (Yawata et al., 2001), but no other close homologues in either mouse or human. We therefore believe that FLJ10111 is human PAUL. It has been cytogenetically mapped to chromosome 14 (14q11.2-q21.3). Further searches revealed putative orthologues in Fugu (41\% amino acid identity to mouse PAUL) and Drosophila (CG113211; 23\% identity; Fig. 1b). We were unable to identify close relatives of mouse or Drosophila PAUL in C. elegans. We did find similarity to several proteins that contain, like PAUL, a pair of RING domains separated by an IBR domain. This arrangement, which has been called a 'RBR signature' (RING-Between RINGS-RING) has been found in both demonstrated and Putative E3 ubiquitin ligases (Marin and Ferrus, 2002). To determine the relationship of PAUL to other RBR proteins, we compared their RING1 and IBR domains. As shown in Fig. 1c and d, PAUL is most similar to Ariadne, which binds E2 ubiquitin conjugating enzymes (Ardley et al., 2001), and is also closely related to Parkin, which has been shown to function as an E3 ubiquitin ligase (Zhang et al., 2000). These relationships strengthen the argument that PAUL is an E3 ubiquitin ligase.

\subsection{Interaction of PAUL and MuSK}

PAUL was isolated by virtue of its interaction with the cytoplasmic domain of MuSK in the yeast two-hybrid assay. Further assays in yeast showed that PAUL bound to a fragment containing the carboxy-terminal 140 aa of MuSK's 350 aa-long cytoplasmic domain, indicating that the amino-terminal 210 aa were dispensable for this interaction (data not shown). Interacting PAUL cDNA C13 showed that its RING and IBR domains were dispensable for interaction with MuSK (Fig. 1b). Thus, sites in the amino-terminal portion of PAUL bind sites in the carboxyterminal portion of the MuSK cytoplasmic domain.

Association of MuSK and PAUL in the cytoplasm of mammalian cells was demonstrated by co-transfecting cDNAs encoding epitope-tagged derivatives of both proteins into a human embryonic kidney (HEK-293-T) cell line, immunoprecipitating with an antibody to one member of the pair, and immunoblotting the resulting complexes with an antibody to the other member of the pair. Following transfection with myc-PAUL and GFP-MuSK, immunoprecipitates collected with anti-myc contained GFP-MuSK; no GFP-MuSK was present in control precipitates (Fig. 2a). Likewise, following transfection with FLAG-tagged MuSK (a)

\section{IP: myc \\ IB: MuSK}
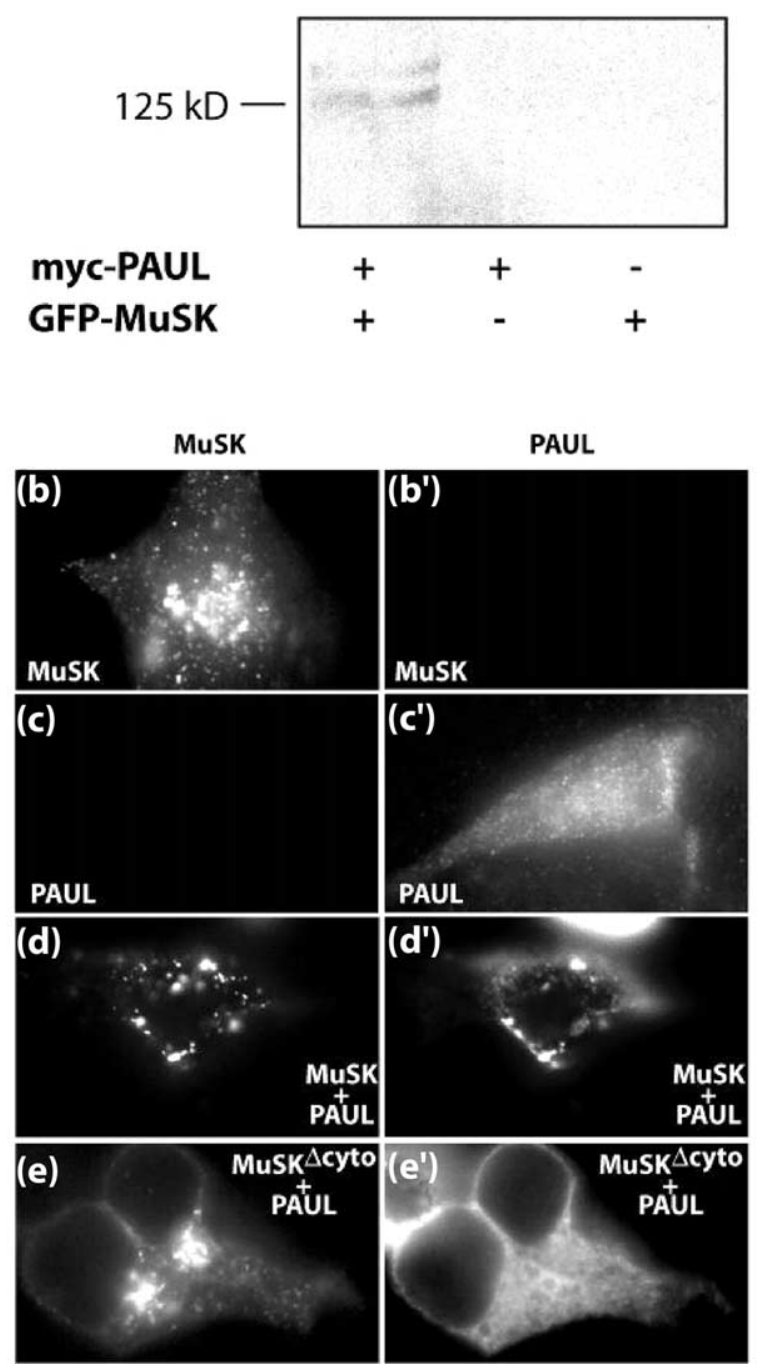

Fig. 2. Association of PAUL and MuSK in transfected cells. (a) HEK-293-T cells were transfected with myc-PAUL, GFP-MuSK, or both, as indicated. Myc-PAUL was immunoprecipitated from cell lysates using myc antibody (9E10), then precipitates were resolved by electrophoresis and probed with anti-MuSK. GFP-MuSK is co-immunoprecipitated with myc-PAUL. (b-d) HEK-293-T cells were transfected with GFP-MuSK (b, $\left.b^{\prime}\right)$, myc-PAUL $\left(\mathrm{c}, \mathrm{c}^{\prime}\right)$, or both $\left(\mathrm{d}, \mathrm{d}^{\prime}\right)$, then permeabilized and stained with anti-myc. Coexpression of MuSK redistributes PAUL from the cytosol to MuSK-rich, membrane-associated puncta. (e, $\left.\mathrm{e}^{\prime}\right)$ A mutant MuSK lacking almost the entire cytoplasmic domain still localizes to puncta within the plasma membrane, but fails to recruit PAUL to the membrane.

and PAUL, immunoprecipitates collected with anti-FLAG contained PAUL (data not shown).

To examine the consequences of MuSK-PAUL interaction on their localization, we stained HEK-293-T cells following transfection with myc-PAUL and/or GFP-MuSK. MuSK was localized to the membrane and to small, intracellular, presumably vesicular puncta (Fig. 2b). In contrast, in cells transfected with myc-PAUL alone, PAUL was present throughout the cytoplasm (Fig. 2c). 
Co-expression of both constructs had no apparent effect on localization of MuSK, but led to redistribution of most of the PAUL to MuSK-positive membrane-associated patches (Fig. 2d). A similar redistribution of PAUL occurred when it was co-expressed with a mutant of MuSK previously demonstrated to lack kinase activity $\left(\mathrm{MuSK}^{\mathrm{K} 608 \mathrm{~A}}\right.$; Glass et al., 1997; data not shown). In contrast, no redistribution of PAUL occurred when it was co-expressed with a MuSK mutant lacking its cytoplasmic domain (GFP-MuSK ${ }^{\Delta \text { cyto; }}$ Zhou et al., 1999), even though the MuSK mutant itself formed membrane-associated patches (Fig. 2e). These data suggest that MuSK can recruit PAUL to the membrane.

\subsection{Distribution of PAUL $m R N A$}

We first used Northern blotting to assess expression of PAUL. This analysis showed the presence of a $\sim 4 \mathrm{~kb}$ PAUL RNA, consistent with the sequence assembled from cDNA cloning. This RNA species was detected in all adult tissues tested, including skeletal muscle and brain, although its abundance varied among tissues (Fig. 3a). PAUL RNA was also present in brain from a postnatal day $(\mathrm{P})$ two mouse.

To localize sites of PAUL expression in embryonic tissue, we used non-radioactive in situ hybridization. PAUL was expressed in many tissues at embryonic day (E) 17, but its level varied considerably among tissues (Fig. 4). Particularly high levels of expression were detected in the brain, liver, kidney and thymus (Figs. 4a,5a,g-i). Expression was also detected in muscle, but the apparent abundance of PAUL RNA was lower in muscles than in many other organs, a pattern different from that observed in adult tissues, as determined by Northern analysis.

Within the developing nervous system both progenitor cells in proliferative zones and postmitotic cells in neuron-rich layers expressed PAUL (Fig. 5b-d). These results raised the possibility that PAUL is expressed in neurons. To address this issue, we examined the distribution of PAUL RNA in adult brain. Expression persisted in several neuronal population in the adult, with notably high levels in the granule and pyramidal cells of the hippocampus (Fig. 5e) and scattered neurons in deep layers of the cerebral cortex (Fig. 5f).

\subsection{Distribution and regulation of PAUL protein}

To assay the distribution of PAUL protein, we generated a rabbit antiserum to a recombinant fragment comprising the carboxy-terminal half of PAUL. Immunoblotting of cells transfected with a cDNA encoding full-length PAUL showed that the resulting antibodies specifically recognized a protein of $\sim 135 \mathrm{kD}$ (Fig. 3b); this size is consistent with, though slightly larger than, that predicted from the cDNA sequence. A protein of similar size was detected in all adult tissues tested, with highest levels in brain and lung (Fig. 3c). A second immunoreactive species of $\sim 115 \mathrm{kD}$, detected in brain and kidney may a represent smaller isoform generated, for example, by alternative splicing.
However, because a similar band was present in cells transfected with a single cDNA, we favor the idea that the smaller band results from proteolytic degradation, either in cells or during sample preparation.

To determine whether PAUL is developmentally regulated, we compared levels of PAUL protein in tissues from neonatal (P0) and adult tissues (Fig. 3d). In skeletal and cardiac muscles, PAUL levels were several-fold higher at P0 than in adults. In contrast, levels of PAUL in lung and brain varied only a few-fold or less between neonates and adults. Given the developmental decrease observed in muscle, we also asked whether PAUL levels increased following denervation, as has been reported for several components of the postsynaptic membrane at the NMJ, including AChRs and MuSK (Bowen et al., 1998; Sanes and Lichtman, 1999). However, levels of PAUL in 3- or 10-day denervated muscles were not significantly different than those in control, innervated muscles (data not shown).

Finally, in view of the association of PAUL with MuSK in vitro, we used two methods to ask whether PAUL is
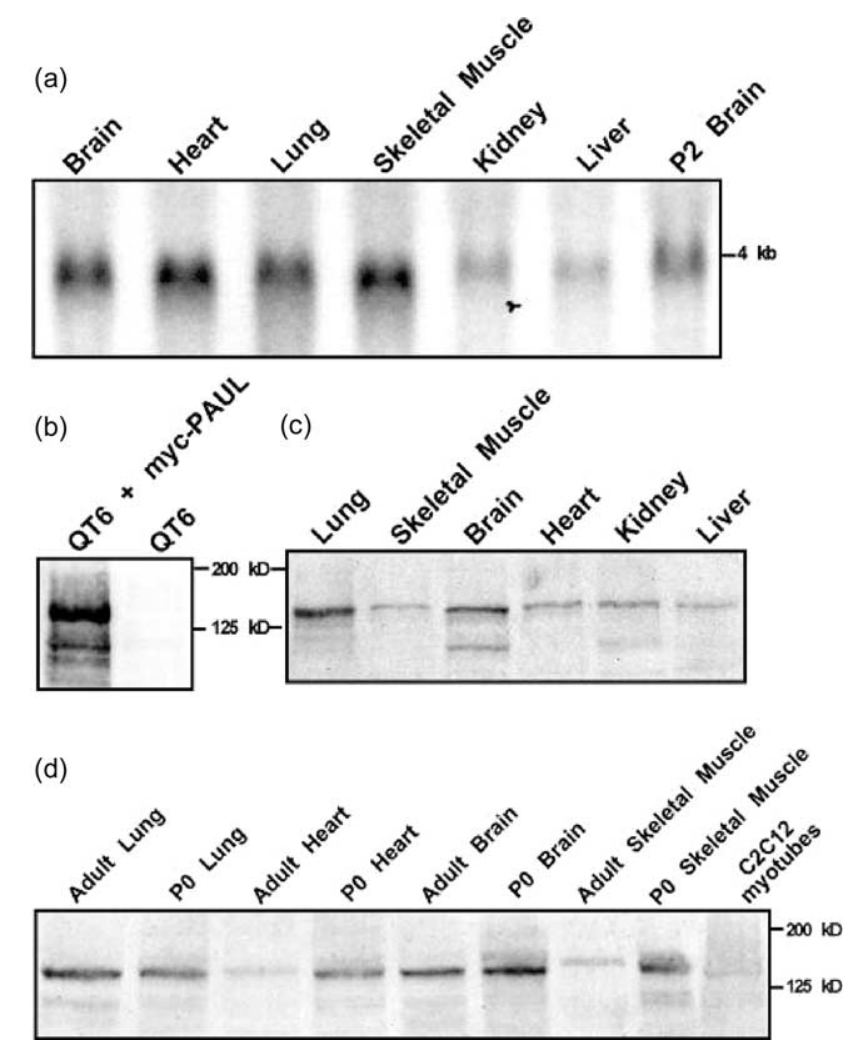

Fig. 3. Detection and regulation of PAUL RNA and protein. (a) Northern analysis of PAUL RNA. A 4 kb RNA is detectable in multiple adult mouse tissues and in P2 mouse brain. (b) On immunoblots, antiserum to PAUL specifically recognizes a $135 \mathrm{kD}$ protein in PAUL-transfected QT6 cells. (c) Western analysis of tissues from adult mice using anti-PAUL. A $135 \mathrm{kD}$ protein band is detectable in all tissues tested, with highest levels in lung and brain. (d) Comparison of PAUL levels in P0 and adult tissues. PAUL levels decrease in cardiac and skeletal muscle during postnatal development. PAUL is barely detectable in cultured myotubes differentiated from the myogenic $\mathrm{C} 2 \mathrm{C} 12$ cell line. 


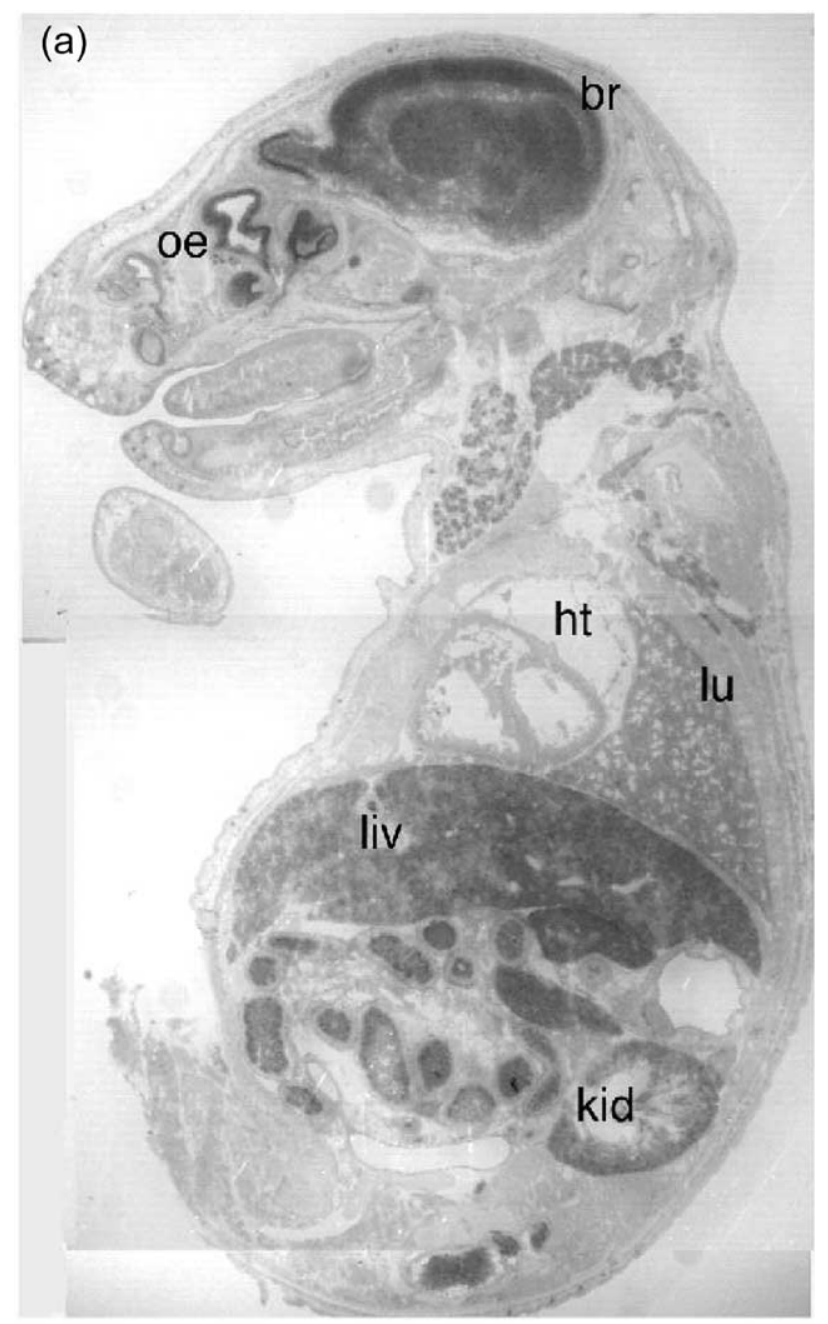

(b)

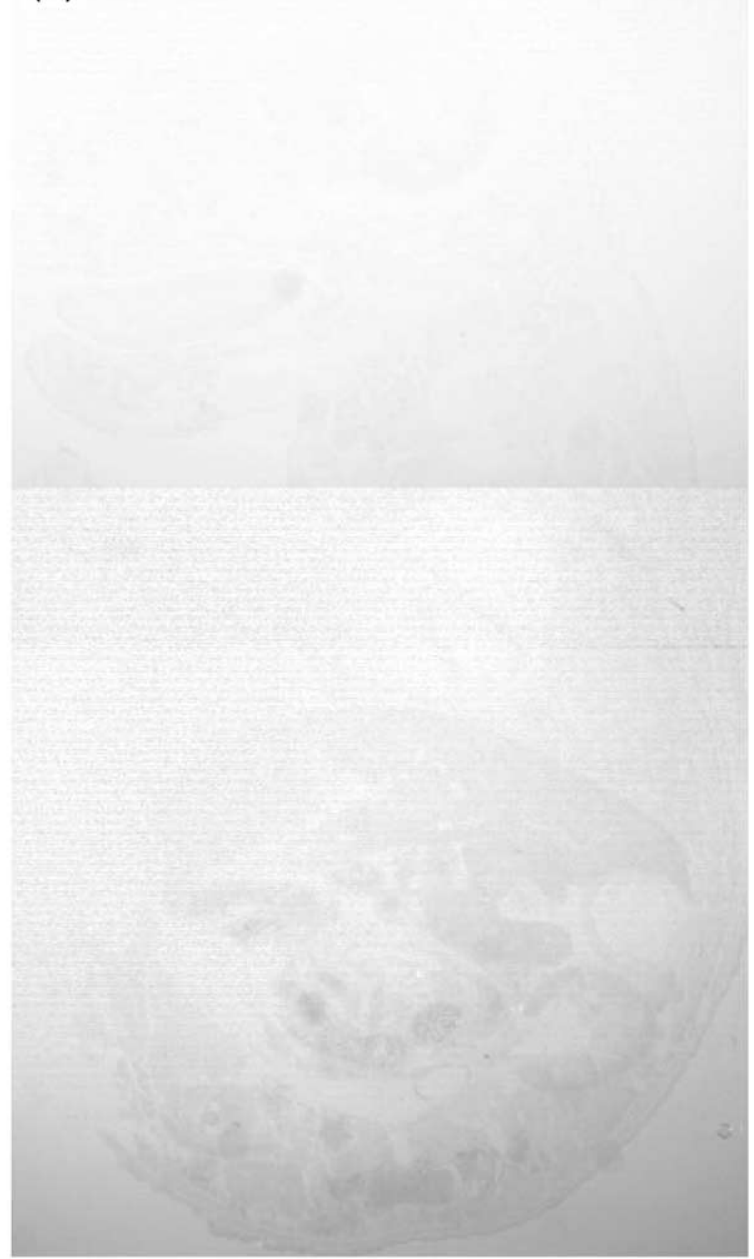

Fig. 4. Expression of $P A U L$ in the mouse embryo (a) Saggital section of an E17 mouse embryo. As assessed by in situ hybridization, expression of $P A U L$ is broad, but its abundance varies among tissues. (b) Adjacent section hybridized and stained in parallel with a control (sense) probe. br, brain; ht, heart; kid, kidney; liv, liver; lu, lung; oe, olfactory epithelium.

selectively associated with the postsynaptic apparatus. First, sections of E18 muscles were doubly stained with antiPAUL plus rhodamine-labeled $\alpha$-bungarotoxin, which specifically labels the AChRs at synaptic sites. PAUL immunoreactivity was detectable in myotubes, but not in the connective tissue cells that separate fascicles of myotubes (Fig. 6a,b). PAUL was present at bungarotoxin-labeled synaptic sites, but was not confined to them (Fig. 6c, $\mathrm{c}^{\prime}$ ). In adult muscle, PAUL levels were barely detectable, consistent with Western blotting data (Fig. 3d), and again no enrichment was observed at synaptic sites (data not shown). Second, we stained C2C12 myotubes, in which AChRs form distinct clusters following treatment with the nerve-derived synaptic organizing molecule, agrin (Sanes and Lichtman, 1999). Because C2C12 cells express only low amounts of PAUL (Fig. 3d), we transfected myoblasts with myc-tagged PAUL before fusion to form myotubes, then stained with anti-myc and rhodamine-labeled $\alpha$-bungarotoxin. As observed in vivo, PAUL was present throughout the cytoplasm of the myotubes, in both receptor-rich and receptor-poor areas, but it was not selectively localized to areas of high receptor density (Fig. 6d).

\section{Experimental procedures}

\subsection{Isolation and sequence analysis of paul}

An E17 mouse cDNA library was screened using the cytoplasmic domain of MuSK as bait. The screen was performed in the HF7c yeast strain harboring HIS3 and $\beta$-gal reporter genes under control of GAL4-binding sites. Details of the screen were described previously (Apel et al., 2000). PAUL cDNAs isolated from the library were sequenced, and the sequence was extended by RACE using poly $(\mathrm{A})^{+}$selected RNA from mouse adult brain. Protein sequence motifs were detected using BLAST and PFAM search engines. 

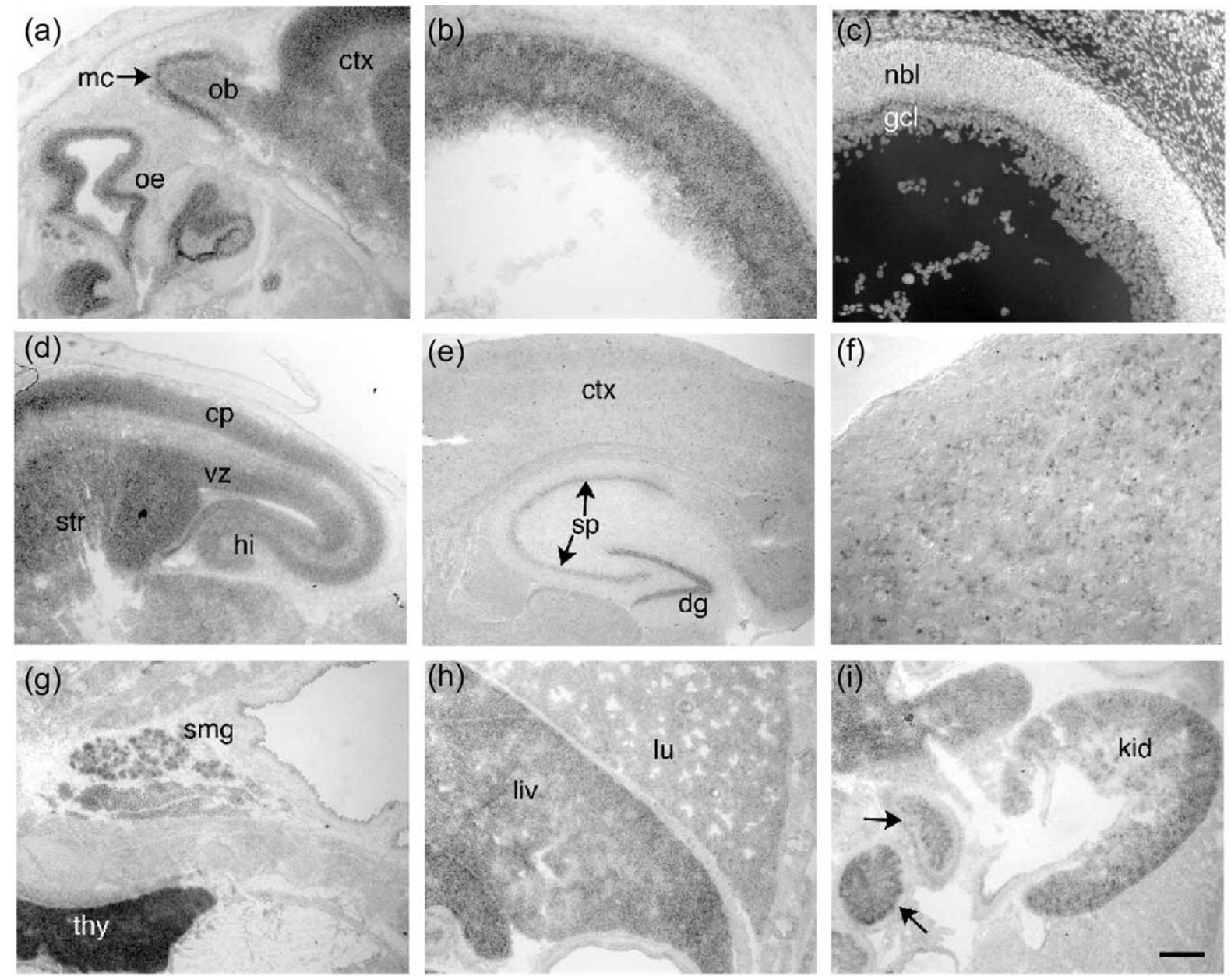

Fig. 5. Distribution of PAUL RNA in neural and non-neural tissues (a) High power micrographs of sections from E17 and adult mice. Olfactory system at E17. PAUL is expressed in cells of the olfactory epithelium (oe), olfactory bulb (ob), and rostralcortex (ctx), with high levels in mitral cells (mc) of the bulb. (b,c) E17 retina shows labeling throughout the neuroblast (nbl) and nascent ganglion cell layers (gcl) ((c) shows nuclei, stained with DAPI, in same field as (b)). (d) E17 brain showing expression in the cortical ventricular zone (vz), cortical plate (cp), striatum (str), and hippocampus (hi). (e) Adult hippocampus shows persistent expression in signal in the dentate gyrus (dg) and stratum pyramidale (sp). (f) Adult cortex, showing scattered PAUL-positive cells. The pial surface is at the top. (g) E17 neck, showing strong expression in submandibular gland (smg) and thymus. (h) E17 lung (lu) and liver (liv). (i) E17 kidney (kid) and gut endothelia (arrows).

Protein sequence alignments were performed using MegAlign software (DNA Star). Genbank accession numbers are as follows: mouse PAUL (AY283085), human PAUL (NP_060469), Fugu PAUL (Scaffold_876), Drosophila PAUL (AAF52435), human novel IBR protein (CAB92088, C. elegans Ariadne-2 (Q22431), Drosophila Ariadne-2 (NP_477374), mouse Ariadne-2 (NP_035920), mouse Ariadne-1 (XP_134935), Drosophila Ariadne-1 (NP_523399), mouse Parkin (NP_057903), human ARA54 (NP_004281).

\subsection{Generation of antibodies}

A segment of mouse PAUL corresponding to the carboxy-terminal 333 amino acids was expressed as a glutathione-S-transferase (GST) fusion protein in bacteria. Partially purified protein obtained by preparative gel electrophoresis of bacterial inclusion bodies was used to immunize rabbits.

\subsection{RNA and protein analysis}

For Northern analysis, poly $(\mathrm{A})^{+}$RNA was prepared from mouse tissues, fractionated on formaldehyde-agarose gels and transferred to GeneScreen Plus membrane (Perkin Elmer, Boston, MA). Blots were probed with ${ }^{32} \mathrm{P}$-labeled PAUL cDNA probe (random primed DNA labeling kit; Roche Molecular Biochemicals, Indianapolis, IN).

For protein analysis, tissues were ground in solubilization buffer (PBS, 1\% Triton X-100, $100 \mu \mathrm{M}$ PMSF, and protease inhibitor cocktail) and centrifuged at $13,000 \mathrm{~g}$ for $30 \mathrm{~min}$ at $4{ }^{\circ} \mathrm{C}$. Protein concentration of supernatants was determined by BCA assay using BSA as standard, and aliquots corresponding to $100 \mu \mathrm{g}$ protein were resolved by gel electrophoresis, transferred to nitrocellulose 

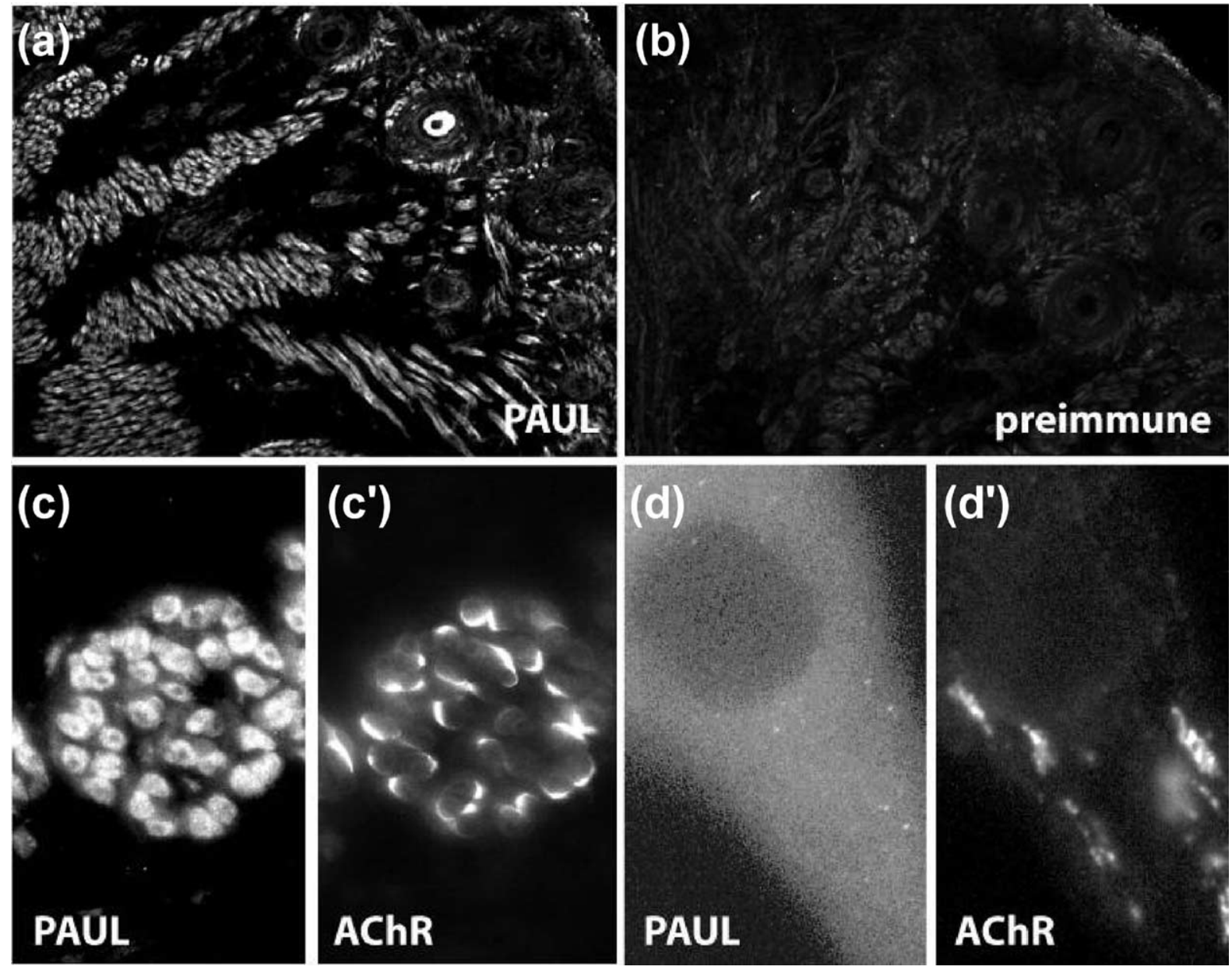

Fig. 6. Cellular distribution of PAUL in skeletal myotubes. (a-c) Cryostat sections of E18 mice were stained with anti-PAUL (a and c) or preimmune rabbit serum (b). The section in (c) was counterstained with bungarotoxin to label synaptic sites ( $\mathrm{c}^{\prime}$ ). Skeletal myotubes are labeled by anti-PAUL; immunoreactivity is present at synaptic sites but is not concentrated at them. $\left(\mathrm{d}, \mathrm{d}^{\prime}\right) \mathrm{C} 2 \mathrm{C} 12$ myotube transfected with myc-PAUL, then labeled with anti-myc and bungarotoxin. As in vivo, PAUL is present but not concentrated in receptor-rich areas.

membranes, and probed with anti-PAUL. For immunoprecipitation, HEK-293-T, quail fibroblast (QT6) or mouse myogenic (C2C12) cells were obtained from ATCC, transfected using Fugene6 reagent (Roche), then harvested one day later. Cells were lysed in solubilization buffer (PBS, $1 \%$ Triton X-100, 1\% BSA, $1 \mathrm{mM}$ iodoacetamide, $1 \mathrm{mM}$ PMSF, and protease inhibitor cocktail). Cell extracts were centrifuged at $13,000 \mathrm{~g}$ at $4{ }^{\circ} \mathrm{C}$ for $30 \mathrm{~min}$, and then supernatants were subjected to immunoprecipitation with antibodies to c-myc (9E10) or anti-FLAG epitope tag (M2, Sigma, St Louis, MO). Complexes were isolated by binding to protein G-Sepharose (Amersham Biosciences, Piscataway, NJ) and resolved by electrophoresis and immunoblotting.

\subsection{Histology}

Tissues were fixed in PBS-buffered $4 \%$ paraformaldehyde (PFA), dissected, infiltrated with sucrose, frozen, and sectioned in a cryostat at $8 \mu \mathrm{m}$. Sections were incubated with anti-PAUL in blocking buffer (PBS, 4\% BSA, $0.1 \%$ Triton X-100), then washed extensively with PBS and incubated for $1 \mathrm{~h}$ at room temperature with a mixture of rhodamine-labeled $\alpha$-bungarotoxin and Alexa-488 conjugated anti-rabbit IgG. HEK-293-T or C2C12 cells were fixed for $20 \mathrm{~min}$ at room temperature with PBS-buffered $2 \%$ PFA, then stained as above. $\mathrm{C} 2 \mathrm{C} 12$ cells were maintained in fusion medium (DMEM plus $2 \%$ horse serum) for 5 days to form multinucleated myotubes prior to staining.

In situ hybridization was performed using digoxigeninlabeled riboprobes, as described by Yin et al. (2002).

\section{Acknowledgements}

This work was supported by grants from the N.I.H. to J.R.S. 


\section{References}

Apel, E.D., Lewis, R.M., Grady, R.M., Sanes, J.R., 2000. Syne-1, a dystrophin- and Klarsicht-related protein associated with synaptic nuclei at the neuromuscular junction. J. Biol. Chem. 275, 31986-31995.

Ardley, H.C., Tan, N.G., Rose, S.A., Markham, A.F., Robinson, P.A., 2001. Features of the parkin/ariadne-like ubiquitin ligase, HHARI, that regulate its interaction with the ubiquitin-conjugating enzyme, Ubch7. J. Biol. Chem. 276, 19640-19647.

Bowen, D.C., Park, J.S., Bodine, S., Stark, J.L., Valenzuela, D.M., Stitt, T.N., Yancopoulos, G.D., Lindsay, R.M., Glass, D.J., DiStefano, P.S., 1998. Localization and regulation of MuSK at the neuromuscular junction. Dev. Biol. 199, 309-319.

DiAntonio, A., Fetter, R.D., Bergstrom, K., Strauss, R., Goodman, C.S., 2000. Ubiquitination-dependent mechanisms regulate synaptic growth and function. Neuron 26, 313-329.

Ehlers, M.D., 2003. Activity level controls postsynaptic composition and signaling via the ubiquitin-proteosome system. Nat. Neurosci. 6, $231-242$.

Glass, D.J., 2003. Signalling pathways that mediate skeletal muscle hypertrophy and atrophy. Nat. Cell Biol. 5, 87-90.

Glass, D.J., Bowen, D.C., Stitt, T.N., Radziejewski, C., Bruno, J., Ryan, T.E., Gies, D.R., Shah, S., Mattsson, K., Burden, S.J., DiStefano, P.S., Valenzuela, D.M., DeChiara, T.M., Yancopoulos, G.D., 1996. Agrin acts via a MuSK receptor complex. Cell 85, 513-523.

Glass, D.J., Apel, E.D., Shah, S., Bowen, D.C., DeChiara, T.M., Stitt, T.N., Sanes, J.R., Yancopoulos, G.D., 1997. Kinase domain of the musclespecific receptor tyrosine kinase (MuSK) is sufficient for phosphorylation but not clustering of acetylcholine receptors: required role for the MuSK ectodomain? Proc. Natl. Acad. Sci. USA 94, 8848-8853.

Hegde, A.N., DiAntonio, A., 2002. Ubiquitin and the synapse. Nature 3, $852-861$.

Hofmann, K., Bucher, P., 1996. The UBA domain: a sequence motif present in multiple enzyme classes of the ubiquitination pathway. Trends Biochem. Sci. 21, 172-173.
Joazeiro, C.A., Weissman, A.M., 2000. RING finger proteins: mediators of ubiquitin ligase activity. Cell 102, 549-552.

Keller, S.H., Lindstrom, J., Ellisman, M., Taylor, P., 2001. Adjacent basic amino acid residues recognized by the COP I complex and ubiquitination govern endoplasmic reticulum to cell surface trafficking of the nicotinic acetylcholine receptor $\alpha$-subunit. J. Biol. Chem. 276, 18384-18391.

Marin, I., Ferrus, A., 2002. Comparative genomics of the RBR family, including the Parkinson's disease-related gene parkin and the genes of the ariadne subfamily. Mol. Biol. Evol. 19, 2039-2050.

McMahan, U.J., 1990. The agrin hypothesis. Cold Spring Harbor Symp. Quant. Biol. 55, 407-418.

Pride, Y.B., Quinnan, L.R., Verma, S., Malouf, N.A., Husson, H., Salgia, R., Lipkowitz, S., Griffin, J.D., de Melker, A.A., 2002. c-Cbl ubiquitinates the EGF receptor at the plasma membrane and remains receptor associated throughout the endocytic route. Oncogene 21, $1423-1433$.

Sanes, J.R., Lichtman, J.W., 1999. Development of the vertebrate neuromuscular junction. Annu. Rev. Neurosci. 22, 389-442.

Sanes, J.R., Lichtman, J.W., 2001. Induction, assembly, maturation and maintenance of a postsynaptic apparatus. Nat. Rev. Neurosci. 2, 791-805.

Shenoy, S.K., McDonald, P.H., Kohout, T.A., Lefkowitz, R.J., 2001. Regulation of receptor fate by ubiquitination of activated $\beta_{2}$-adrenergic receptor and $\beta$-arrestin. Science 294, 1307-1314.

Yawata, M., Murata, S., Tanaka, K., Ishigatsubo, Y., Kasahara, M., 2001. Nucleotide sequence analysis of the approximately $35-\mathrm{kb}$ segment containing interferon-gamma-inducible mouse proteasome activator genes. Immunogenetics 53, 119-129.

Yin, Y., Miner, J.H., Sanes, J.R., 2002. Laminets: laminin- and netrinrelated genes expressed in distinct neuronal subsets. Mol. Cell. Neurosci. 19, 344-358.

Zhang, Y., Gao, J., Chung, K.K., Huang, H., Dawson, V.L., Dawson, T.M., 2000. Parkin functions as an E2-dependent ubiquitin- protein ligase and promotes the degradation of the synaptic vesicle-associated protein, CDCrel-1. PNAS USA 97, 13354-13359.

Zhou, H., Glass, D.J., Yancopoulos, G.D., Sanes, J.R., 1999. Distinct domains of MuSK mediate its abilities to induce and to associate with postsynaptic specializations. Cell Biol. 146, 1133-1146. 